\title{
Exploratory Study of knowledge and experience of Polycystic Ovary Syndrome (PCOS) among PCOS-diagnosed Bangladeshi women
}

\section{Ruhun Wasata $^{1} *$, Ilana R. A. Chertok ${ }^{2}$, Caroline Kingori ${ }^{3}$, and Zelalem T. Haile ${ }^{4}$}

${ }^{1}$ Senior Lecturer, Department of Social Relations, East West University, Dhaka, Bangladesh

${ }^{2}$ Professor and Associate Director of Nursing Research and Scholarship, School of Nursing, College of Health Sciences and Professions, Ohio University, Athens, OH, United States

${ }^{3}$ Associate Professor, Department of Social and Public Health, College of Health Sciences and Professionals, Ohio University, Athens, OH, United States

${ }^{4}$ Assistant Professor, Department of Social Medicine, Heritage College of Osteopathic Medicine, Ohio University, Dublin, OH, United States

*Corresponding author: Ruhun Wasata, Senior Lecturer, Department of Social Relations, East West University, Dhaka, Bangladesh..

Received date: February 12, 2020; Accepted date: February 27, 2020; Published date: March 02,2020

Citation: Wasata R, Ilana R. A. Chertok, Kingori C, and Zelalem T. Haile, (2020) Exploratory study of knowledge and experience of Polycystic Ovary Syndrome (PCOS) among PCOS-diagnosed Bangladeshi women. J Women Health Care Issues, 3(1); Doi:10.31579/2642-9756/021

Copyright: (C) 2020 Ruhun Wasata, This is an open access article distributed under the Creative Commons Attribution License, which permits unrestricted use, distribution, and reproduction in any medium, provided the original work is properly cited.

\section{Abstract}

Objective: To explore the perceived experience of polycystic ovary syndrome (PCOS) among PCOS-diagnosed Bangladeshi women with examination of the influence of culture.

Methods: This qualitative study included 25 Bangladeshi women who had been diagnosed with PCOS. The qualitative interviews were completed via Skype and/ or phone between December 2017 to March 2018. Transcriptions of recorded, deidentified responses were analyzed using qualitative manual coding and thematic analysis.

Results and discussion: Three overarching themes were identified from the narratives: PCOS diagnosis experience, PCOS knowledge, and role of religion and culture in relation to PCOS. The women expressed an unsatisfactory experience with their PCOS diagnosis including having to change healthcare professionals, inadequate access to resources, and concerns related to infertility and pregnancy complications. Lack of available evidence-based information was associated with limited knowledge of PCOS among the PCOS-diagnosed Bangladeshi women and their families. The respondents perceived that culture influenced refraining from sharing of information about PCOS thereby limiting knowledge, muting women's health experience, and contributing to a negative perception on PCOS and related conditions. Participants felt that cultural expectations and emphasis on childbearing placed constant pressure on women and stigmatized PCOS.

Conclusions: Bangladeshi women with PCOS perceive various challenges and limited access to information and resources. To minimize some of the challenges associated with PCOS, public health efforts should focus on increasing awareness, education, and resources for women and their families.

Keywords: PCOS; bangladeshi women; PCOS diagnosis; PCOS experiences; PCOS knowledge; culture and PCOS

Abbreviations: PCOS: Polycystic Ovary Syndrome

\section{Introduction}

Polycystic ovary syndrome (PCOS), the most common female endocrine disorder, is estimated to affect $8-20 \%$ women of reproductive age worldwide $[1,2,3,4,5,6]$, with rates in the Indian subcontinent ranging from 5-10\% and a noted increasing prevalence [2]. Globally, the research on PCOS has been largely focused on the medical aspects of the disorder [3]. Knowledge and education regarding PCOS among the PCOSdiagnosed population and the general population has received limited attention and research focus [3]. As a public health issue, knowledge and education of PCOS has practical implications that impact women and their families and should therefore be included in research and scholarship activities on the topic.

Despite recent medical advances, PCOS is a poorly understood condition [7]. The common signs and symptoms of PCOS include menstrual irregularities (amenorrhea, oligomenorrhea, very heavy periods, anovulatory periods), obesity, pelvic pain, insulin resistance, infertility, excessive hair growth (hirsutism), severe acne, skin problems, oily skin, and patches of thickened, dark, velvety skin (acanthosis nigricans) [1, 2, $5,7]$. The symptoms of PCOS vary between women and may change over time. Different conditions have been associated with PCOS and often been identified as results of PCOS; those conditions include but are not limited to: obstructive sleep apnea, dry eye syndrome, cardiovascular disease, high cholesterol, fatigue, endometrial cancer, and psychological 
problems including eating disorders (anorexia, bulimia, and binge eating), depression, anxiety, and mood-swings [1, 5, 6, 7, 8, 9]. PCOS has been identified as the most common cause of anovulatory infertility or subfertility among women worldwide [10]. Additionally, women with PCOS have two to four times higher risk of developing metabolic complications than that of women without PCOS and increased risk of developing type 2 diabetes $[6,8,11]$. Evidently, having PCOS can negatively affect women's quality of life (QOL) $[6,8,11,13]$.

A recent study conducted by a leading Medical University in Bangladesh found that prevalence rates of PCOS among women of reproductive age in Bangladesh have been increasing [13], elevating the need for concern and address of women in this country. To date, studies conducted on PCOS in Bangladesh have focused largely on clinical and biomedical aspects of PCOS, not on knowledge and perception of PCOS. The lack of understanding of the knowledge and perception on PCOS among women and their healthcare providers poses challenges to the delivery of accurate information and consistent evidence-based PCOS management [14]. Examination of the knowledge and perception of PCOS among women in Bangladesh is necessary to inform the development of educational programs. This research is the first study to capture the experience and knowledge of PCOS-diagnosed women in Bangladesh.

\section{Materials and methods}

\subsection{Study setting and design overview}

A qualitative approach using a qualitative checklist that was developed based on a study of women with PCOS by Avery and Braunack-Mayer (2007). With the authors' permission, their original work was adopted and modified. Institutional review board approval was granted by the researchers' academic institution.

\subsection{Sample population}

This qualitative portion of the study is part of a larger research study. For the qualitative interviews, 25 women were recruited from among participants who answered the web-based quantitative survey between December 2017 and March 2018. The survey had been shared via social media platform. The inclusion criteria for participation were Bangladeshi women aged 18 to 45 years and previously diagnosed with PCOS. Participation was voluntary, after written, emailed consent, and women did not receive any financial benefit for study participation.

\subsection{Data collection and analysis}

Upon completion of the anonymous questionnaire, participants were asked about their willingness to participate in the qualitative part of the research. Participants who expressed their interest in participating in the qualitative interview via Skype and/or phone and submitted their contact information were contacted by the researcher. Nearly all of the interviews were conducted in the native Bengali language with one interview completed in English. The interviews were transcribed and translated into English and validated by blinded native Bengali speakers. To maintain confidentiality, identifying information was removed from the data.

To describe the participants' demographic information, a frequency analysis was conducted. For the qualitative data, the translated data was color-coded manually and reorganized based on themes. Three themes were identified: (1) PCOS experience, (2) PCOS knowledge, and (3) the role of culture in relation to PCOS. Under each theme, there were an average of ten to twelve codes identified which were commonly mentioned by the participants. The codes were kept when they were expressed by a minimum of eight participants.

\section{Demographic information of the sample}

Among the 25 interview participants, 22 persons were followers of Islam, all the women were born and raised in Bangladesh, the mean age was 26.7+/-4.9 years, and other characteristics are described in Table 1. Among the eight women who have been trying to conceive, $72.7 \%$ represent married women. Regarding Body Mass Index (BMI) measures $\left(\mathrm{kg} / \mathrm{m}^{2}\right)$, only 11 who represent less than half of the women belonged to the normal weight BMI group $(18.5-24.9)$ and the remaining 14 belonged to the overweight and obese groups.

\begin{tabular}{|c|c|}
\hline Demographic characteristics & $\%$ of women (number) \\
\hline \multicolumn{2}{|l|}{ Age } \\
\hline $18-25$ & $52(13)$ \\
\hline $26-35$ & $40(10)$ \\
\hline $36-45$ & $8(2)$ \\
\hline \multicolumn{2}{|l|}{ Marital Status } \\
\hline Married & $44(11)$ \\
\hline Divorced & $4(1)$ \\
\hline Never married/single & $52(13)$ \\
\hline \multicolumn{2}{|l|}{ Fertility Status } \\
\hline Have been trying to conceive & $32(8)$ \\
\hline Have not been trying to conceive & $68(17)$ \\
\hline \multicolumn{2}{|l|}{ Body Mass Index (BMI) $\left(\mathrm{kg} / \mathrm{m}^{2}\right)$} \\
\hline Underweight: $<18.5$ & $0(0)$ \\
\hline Normal weight: $18.5-24.9$ & $44(11)$ \\
\hline Overweight: $25-29.9$ & $28(7)$ \\
\hline Obese: 30 or more & $28(7)$ \\
\hline
\end{tabular}

Table 1. Characteristics of the participating Bangladeshi women with PCOS $(n=25)$. 
Only two participants received their PCOS diagnosis in less than a year whereas the highest number of women (10) waited five or more years to be diagnosed, and the remaining 13 respondents waited varying times ranging from two to four years (Table 2). Only eight participants were diagnosed with PCOS by the first healthcare provider they visited, while the majority of women needed to change healthcare provider multiple times to be diagnosed. Most of the women (21) reported visiting gynecologists for diagnosis of their symptoms. Additionally, nine participants sought treatment from endocrinologists, five from homeopathic doctors, and four from other professionals including nutritionists. Over half of the participants were diagnosed when they were at least 21 years of age. Regarding symptoms associated with PCOS which prompted the women to visit their healthcare providers, 17 went for menstrual problems, 12 went for issues regarding overweight or problems with weight control despite diet and exercise, 14 went for depression, 11 for unpredictable mood swings, nine for anxiety, nine for low/lack of self-esteem, 12 went for excessive hair loss, 11 went for visible growth of hair on face or other parts of the body and eight for severe skin problems.

\begin{tabular}{|c|c|}
\hline & \% of women (number) \\
\hline \multicolumn{2}{|l|}{ Time until diagnosis } \\
\hline Less than a year & $8(2)$ \\
\hline Within $1.1-2.0$ years & $12(3)$ \\
\hline Within $2.1-3.0$ years & $16(4)$ \\
\hline Within $3.1-4.0$ years & $16(4)$ \\
\hline Within $4.1-5.0$ years & $8(2)$ \\
\hline Within 5.1 or more years & $40(10)$ \\
\hline \multicolumn{2}{|l|}{ Number of healthcare professionals visited before diagnosis } \\
\hline $0-1$ & $32(8)$ \\
\hline $2-3$ & $40(10)$ \\
\hline $4-5$ & $24(6)$ \\
\hline 5 and more & $4(1)$ \\
\hline \multicolumn{2}{|l|}{ Type of healthcare professional visited for diagnosis (multiple answers possible) } \\
\hline Gynecologists & $84(21)$ \\
\hline Endocrinologists & $40(10)$ \\
\hline Doctor of Homeopathy & $20(5)$ \\
\hline Others & $16(4)$ \\
\hline \multicolumn{2}{|l|}{ Age in years at the time of PCOS diagnosis } \\
\hline $11-15$ & $4(1)$ \\
\hline $16-20$ & $36(9)$ \\
\hline $21-25$ & $44(11)$ \\
\hline $26-30$ & $16(4)$ \\
\hline \multicolumn{2}{|l|}{ Symptoms for visiting a healthcare professional (multiple answers possible) } \\
\hline Overweight & $48(12)$ \\
\hline Menstrual problems (Irregular/no menstruation/menstrual cramps) & $68(17)$ \\
\hline (Sudden) severe skin or acne problem & $32(8)$ \\
\hline Excessive hair fall & $48(12)$ \\
\hline Growth of visible hair on face or other body parts & $44(11)$ \\
\hline Emotional disturbance - depression & $56(14)$ \\
\hline Emotional disturbance - anxiety & $36(9)$ \\
\hline Emotional disturbance - Low/ lack of self-esteem & $36(9)$ \\
\hline Emotional disturbance - unpredictable mood swings & $44(11)$ \\
\hline
\end{tabular}

Table 2. Women's PCOS diagnosis experience $(n=25)$.

When asked if they were given explanations of the various aspects of PCOS, most of the women responded either "no" or "yes, but inadequate" (Figure 1). 
Explanation on PCOS by healthcare providers during diagnosis

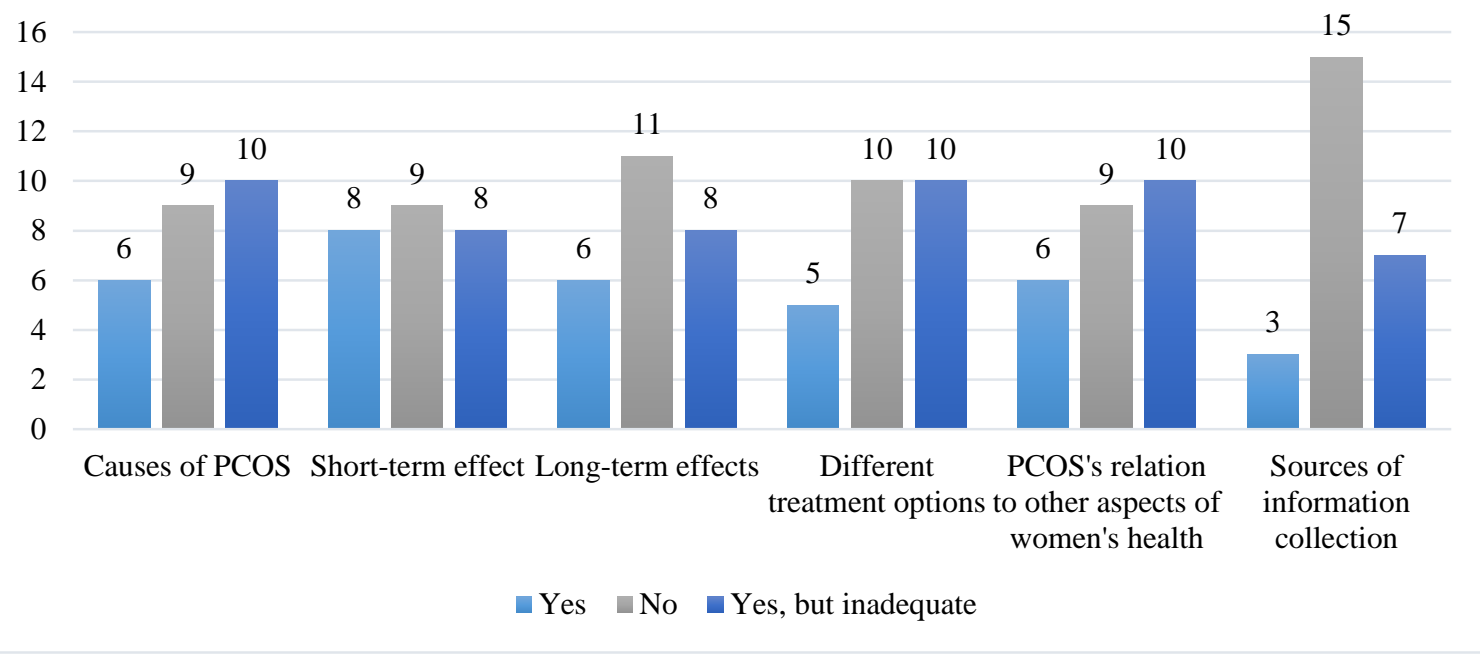

Figure 1. Explanation on PCOS by healthcare providers during diagnosis $(\mathrm{n}=25)$

Regarding knowledge of PCOS, 21 respondents out of 25 respondents reported they knew nothing about PCOS before being diagnosed (Table $3)$. The most common short-term problem identified was related to menstruation and the most common long-term problem was infertility, subfertility, or problems with pregnancy. Table 3 also presents women's knowledge of PCOS management. There was an identified need of more information on PCOS treatment, management, causes, and effects with various options of modes of information delivery. The women primarily discussed PCOS with other females, especially family members and friends. Among the men with whom the women discussed their PCOS were husbands, family members, religious leaders, and spiritual healers.

\begin{tabular}{|c|c|}
\hline & $\%$ of women (number) \\
\hline \multicolumn{2}{|l|}{ Knowledge on PCOS before diagnosis } \\
\hline Nothing & $84(21)$ \\
\hline Related to subfertility/infertility/problems with pregnancy & $16(4)$ \\
\hline \multicolumn{2}{|l|}{ What do you think are the causes of PCOS? (multiple answers possible) } \\
\hline Related to genetics & $44(11)$ \\
\hline Environmental causes & $20(5)$ \\
\hline Related to ovary & $40(10)$ \\
\hline Related to hormones & $68(17)$ \\
\hline Related to insulin & $28(7)$ \\
\hline Causes are yet unknown by the scientists & $16(4)$ \\
\hline I do not know the causes & $16(4)$ \\
\hline Others & $12(3)$ \\
\hline \multicolumn{2}{|l|}{ Identification of the most critical short-term effect of PCOS known by the PCOS diagnosed person. } \\
\hline Period related (irregularities/prolonged/short/bloating during menstruation) & $40(10)$ \\
\hline Weight related (weight gain/overweight/problems to control weight) & $20(5)$ \\
\hline Hair related (excessive hair fall, unwanted hair on different body parts) & $20(5)$ \\
\hline Mood swings & $4(1)$ \\
\hline Others (Skin problems//cysts/cancer of uterus and/or do not know) & $16(4)$ \\
\hline \multicolumn{2}{|l|}{ Identification of the most critical long-term effect of PCOS known by the PCOS diagnosed person. } \\
\hline Infertility/problems with conceiving/ pregnancy complications & $72(18)$ \\
\hline Non-communicable diseases (Cancer of uterus and/or diabetes) & $8(2)$ \\
\hline Problems related to menstruation & $4(1)$ \\
\hline Problems related to hair (excessive hair fall and/or unwanted hair growth on different body parts) & $4(1)$ \\
\hline I do not know & $12(3)$ \\
\hline $\begin{array}{l}\text { Identification of the suitable option for managing or controlling PCOS known by the PCOS } \\
\text { diagnosed person (Multiple answers possible) }\end{array}$ & \\
\hline
\end{tabular}




\begin{tabular}{|c|c|}
\hline Diet chart maintenance without exercise & $16(4)$ \\
\hline Strength training & $12(3)$ \\
\hline Cardio exercise & $8(2)$ \\
\hline Any type of exercise is good & $20(5)$ \\
\hline Exercise with diet chart & $72(18)$ \\
\hline Oral contraceptive pills & $16(4)$ \\
\hline \multicolumn{2}{|l|}{ Information needed on PCOS } \\
\hline Reasons/causes of PCOS & $28(7)$ \\
\hline People at risk/vulnerable population & $4(1)$ \\
\hline Effects of PCOS & $20(5)$ \\
\hline Treatment and management options & $36(9)$ \\
\hline Raising awareness on PCOS & $12(3)$ \\
\hline \multicolumn{2}{|c|}{ Preferred sources of information acquiring } \\
\hline Internet, social media, Facebook, emails & $56(14)$ \\
\hline Electronic and print media, advertisements & $8(2)$ \\
\hline Government & $16(4)$ \\
\hline Doctors and healthcare professionals & $20(5)$ \\
\hline \multicolumn{2}{|c|}{ Discussion on PCOS with different persons/relations: (Multiple answers possible) } \\
\hline Mother & $72(18)$ \\
\hline Father & $16(4)$ \\
\hline Sister(s) & $56(14)$ \\
\hline Brothers & $1(4)$ \\
\hline Husband/Life partner & $60(15)$ \\
\hline Female cousin(s) & $48(12)$ \\
\hline Male cousin(s) & $4(1)$ \\
\hline Female friend(s) & $76(19)$ \\
\hline Male friend(s) & $8(2)$ \\
\hline Female colleague(s) & $40(10)$ \\
\hline Male colleague(s) & $12(3)$ \\
\hline Religious leader(s) (i.e. Imam, Pir, Pundit, Father, Minister) & $4(1)$ \\
\hline Spiritual healer(s) & $8(2)$ \\
\hline
\end{tabular}

\section{Qualitative Results of Thematic Analysis}

\subsection{PCOS diagnosis experience}

Many of the participants reported to be dissatisfied with their PCOS diagnosis experience. For the majority of the respondents, the diagnosis of PCOS required changing more than one healthcare provider within a year. Dissatisfaction was associated with the perceived lack of explanation or information about PCOS and its management. One respondent expressed during her interview:

"...I have been suffering for a long time, I cannot even remember how long. Every year I was changing doctors, taking tests worth of thousands of Taka (Bangladeshi currency). When I was finally diagnosed by a gynecologist, she explained absolutely nothing and gave me medicine which I came to know from pharmacists is used for diabetes. Why would someone give a person of 19 years of age a diabetes medicine when the reports were clearly showing I did not have diabetes? Well, how could I tell? My doctor informed me of nothing..."

Many of the women lacked knowledge of PCOS and its symptoms prior to their diagnosis which contributed to delayed diagnosis and medical management, supporting the findings in Table 3 where 21 respondents reported knowing nothing about PCOS before diagnosis. In the words of one respondent:

"...I waited for long before visiting my doctor. It never occurred to me that perhaps something is wrong with my body. I never heard anything of such kind, otherwise, I would have gone to the doctor earlier..."

The unmarried respondents explained that irregular menstruation or overweight was the main reason for consulting a healthcare provider. 
Young women experiencing irregular menstruation in Bangladesh have often been told that menstruation irregularity would "go away" with age or after marriage, or that conception will help improve their problem and remedy their PCOS.

“...I was a second year bachelor's student (when diagnosed with PCOS). I was not planning to get married very soon, but my doctor emphasized that I should get married very soon and conceive. This is the most important solution. My parents were convinced after hearing that from my doctor and they were constantly pressuring me about that. They still do...".

The married respondents shared that they primarily went to their healthcare providers when they were being unable to conceive. Their experience was perceived by the respondents as feeling frightened and uncertain about their future after learning of their PCOS diagnosis. Much of the feeling of uncertainty was associated with the "inability of becoming pregnant" or "possibility of being infertile," as described by one respondent:

"...I was worried, afraid... I did not know what to expect. The first thing that came to my mind was if I would be able to become a mother or not? My mother was at the doctor's office that day. She expressed the same concern to the doctor..."

Feeling "uncertainty about the future" for the respondents was increased when their family members also expressed almost similar worries about their health and uncertainty of conceiving. Respondents repeatedly expressed that their families, especially their mothers, were concerned about their daughters' ability to become pregnant, as PCOS was not commonly discussed with the women's fathers.

\subsection{Knowledge of PCOS}

Many of the PCOS-diagnosed women reported that they did not know anything about PCOS before being diagnosed and that they continued to have limited knowledge about their condition even after being diagnosed, as shown in Table 3. The issue of limited knowledge by PCOS-diagnosed Bangladeshi women was reflected by one respondent's expression:

“...I knew nothing about PCOS before. I know nothing about PCOS now. My doctor just told me once that I have PCOS, suggested me to lose weight but did not guide me through it. I am maintaining my usual life, trying to eat less. That is all...".

Moreover, the respondents suggested that as they did not get an explanation of how PCOS affected other aspects of their lives including potential emotional turbulence and its management. According to one respondent:

“...I knew that I was going through some emotional changes. Due to lack of awareness, I was never able to relate my emotional disturbance to PCOS. I always thought it was due to all of the things going on in my life. I realized (that PCOS and emotional turbulence) could be related when I took the survey questionnaire for this research which made me Google the symptoms of PCOS. I was surprised to see that I could relate most of the conditions I experienced..."

\subsection{Role of religion and culture in relation to PCOS}

Respondents perceived a strong relationship between Bangladeshi culture, rather than religion, and the lack of knowledge of PCOS. Women's health is regarded as a "secretive issue" in the Bangladeshi culture and that the women tended not to share their health issues with others, particularly males.

"...We never do that. We have been taught throughout our lives that this can never be done; this should never be done. We should keep our menstruation to ourselves, we should keep our pains to ourselves. If someone knows, it is a disgrace for us...".

The women expressed that their health problems had been minimized or dismissed by their healthcare providers and there was an overall lack of discussion about women's health issues, even when the women sought information from professionals. The women had been informed that their PCOS would be "corrected with age and after marriage." This quest for information and dismissive medical response was reflected in the expression of one of the respondents:

“...My mother changed six doctors, all of them suggested that I will be alright when I will grow up, when I will get married, and finally when I will have a baby...".

Another issue identified by the respondents was that there was a cultural component; Bangladeshi women face social pressure from their families to get married, conceive, and give birth. Healthcare providers perpetuate similar attitudes as the women heard messages from them suggesting that getting married and conceiving was the "cure" for PCOS and other gynecological problems. Respondents reported that the healthcare providers played a crucial role in putting pressure on the PCOS-diagnosed women to get married and conceive:

“...The most common suggestion a Bangladeshi woman gets from her gynecologist is to get married and have babies. They do not, I should rather say cannot, consider the idea that a woman perhaps does not want to get married, or wants to get married but does not wish to reproduce. If a woman says anything like that in Bangladesh, she is an anomaly. Pretty soon she is going to experience societal pressure and be ostracized if she does not get married and produce a child before her age expires...".

Respondents reported that cultural factors such as the importance of getting married and having children in combination with limited discussion of women's health and PCOS have caused this condition to be associated with stigma and shame. There is a sense of inappropriateness in discussing PCOS with others as expressed by one of the respondents:

“...You cannot talk to anyone; you cannot inform anyone. I can share a horrifying experience. One of my cousins, from the maternal side, mentioned to her would-be in-laws that she has menstrual irregularities. As a consequence of her honesty, her future possible in-laws called off the wedding. She had to go through severe shaming because of that. Now tell me, who would talk about health if society treated you that way?"

\section{Discussion}

This research study focused on understanding the experience and knowledge of PCOS among PCOS-diagnosed Bangladeshi women. Additionally, this study examined the perceived role of culture in the experience of having PCOS among the respondents. Overall findings are derived from three themes that emerged from the data reflecting the 
experiences of the respondents. The first theme, indicating an overall negative experience with PCOS diagnosis, was found by other researchers $[4,13,14,15]$. Women had to visit multiple healthcare providers over an extensive time to receive a diagnosis of PCOS. This challenge to and dissatisfaction with PCOS diagnosis has been noted in previous studies $[4,13,14,15]$. Changing multiple healthcare providers is associated with the lack of cooperation from healthcare providers and their lack of information and knowledge [4, 13, 14, 15]. Independent of their age, education level, profession, and geographic location, this response was shared by most of the respondents. There is a need for improvement in resources, education, and care of women with PCOS in Bangladesh [4, $13,14,15,16]$.

Lack of knowledge among the healthcare providers [4, 13-15] often resulted in suggesting remedies of PCOS that were not evidence-based such as getting married and conceiving. It is important to give accurate information that PCOS is incurable but is manageable with various treatment options. The lack of knowledge on PCOS among the healthcare providers has been found to result in women's dissatisfaction with their PCOS diagnosis experience [14]. Women desire to be more informed by their healthcare providers of the causes, symptoms, and treatment options of PCOS [4, 13-15]. Lack of information about PCOS among PCOSdiagnosed women can cause increased concern and uncertainty about their future [4, 6-15].

Having limited knowledge of PCOS by the diagnosed women has been found to be a common issue around the world [4, 13-16]. In Bangladesh, important contributing factors regarding the limited knowledge on PCOS include lack of overall health knowledge, lack of proper and accurate PCOS information, lack of support by the healthcare providers in obtaining information, and the consideration of women's health issues as secret topics that should not be discussed [1-4, 6, 13-17]

The most common health concern expressed by the PCOS-diagnosed Bangladeshi women was related to issues of infertility or subfertility associated with PCOS $[14,15,17]$. This concern of fertility problems may overshadow other aspects of PCOS. Married respondents reported seeking treatment for their "infertility" problem while not reporting concerns related to other physical consequences of PCOS [17]. Healthcare providers should educate PCOS-diagnosed women about other major health concerns related to PCOS such as the increased risk of type 2 diabetes, cardiovascular disease, and endometrial cancers [2, 4, 717].

Information availability has a crucial role to play in improving access to knowledge about PCOS among women in Bangladesh [2, 4, 7-17]. The respondents' identification of the Internet as a source of information on PCOS has been found by other researchers $[15,16]$. However, culturally sensitive and inclusive information would benefit Bangladeshi women such as suggestions for dietary modifications and exercise such as strength training [16]. Similarly, strength training is discouraged among women in Bangladesh as it is perceived as masculine. Hence, it is highly recommended that healthcare providers and researchers adapt and convey information on PCOS management in a more culturally sensitive manner for acceptability among Bangladeshi women $[2,4,6$, 7, 12, 14, 15, 16, 17].

The respondents have associated culture as an influencing factor in PCOS knowledge among PCOS-diagnosed Bangladeshi women and healthcare providers [18-23]. The respondents who thought Bangladeshi culture influenced the knowledge of PCOS among Bangladeshi women identified different reasons. A common attitude in the Bangladeshi society is the perception that any disease is negative [18-23]. There is a risk of gossip regarding conditions which are regarded as abnormality, for instance infertility [18-23]. Yet, women's health is generally avoided as subjects of informative discussions [18-23]. Some of the respondents reported discussing PCOS with family and friends, although with preference of discussing their condition only with females. Hence, males are excluded from the conversation on women's health which may limit the dissemination of knowledge on PCOS [18-23].

Bangladeshi women experience pressure related to fertility and childbirth [18-23]. There is a cultural expectation of women to get married and have children [18-23]. Since PCOS is associated with infertility, subfertility, and pregnancy complications, it is difficult for women in this culture to discuss PCOS with other people for fear of stigma. The multiple factors involved contribute to the minimization of the conversation regarding PCOS specifically and women's health in general, further leader to limited knowledge of PCOS among women with the condition in Bangladesh [18-23].

To increase the knowledge of PCOS, it is important to promote PCOS education and public health interventions. Programs to raise awareness are crucial as evident from the research that even the PCOS-diagnosed women and their families lack PCOS knowledge. Public health awareness education of PCOS should involve various stakeholders and agencies to ensure a comprehensive program including the Government of Bangladesh, researchers, healthcare providers, public health practitioners, and PCOS-diagnosed women and their families. Moreover, the incorporation of men in the conversation can help reduce the stigma attached to PCOS in Bangladesh.

Limitations of the study include possible selection bias. Recruitment was done through online methods and snowball sampling among women who have Internet access. Hence, only women with access to the Internet could participate, thereby preventing women living in remote rural areas from participation. Furthermore, there is a possibility of self-report bias and social-desirability bias. As such the results may not be generalizable. Despite these limitations, this is the first study published on the experience of PCOS-diagnosed Bangladeshi women.

Findings from this study can inform further research and the development of programs regarding PCOS among Bangladeshi women. Based on the findings, it is recommended that healthcare providers and public health researchers develop culturally appropriate and effective methods to increase the knowledge and awareness of PCOS among the people of Bangladesh, to eliminate the stigma attached to PCOS, to augment the knowledge and training of healthcare providers regarding resources for managing PCOS, and to avail information and resources regarding PCOS to women and their families in Bangladesh.

\section{Conclusion}

PCOS is a highly complex health issue experienced by women of reproductive age worldwide. Although clinical research studies have been conducted on PCOS in Bangladeshi women, there is a need for an expanded focus on the public health aspect of PCOS. Findings from this qualitative research study identified three themes: PCOS diagnosis 
experience, PCOS knowledge, and role of religion and culture in relation to PCOS. The first theme pointed to dissatisfaction among PCOSdiagnosed women in Bangladesh with their PCOS diagnosis experience. According to the second theme, the diagnosed women lacked knowledge of PCOS, received limited information from their healthcare providers, and felt increased pressure and concern about getting married and having children. The third theme presented a cultural perspective, that women's health is a generally avoided topic of discussion in Bangladesh which further limits women's knowledge of PCOS and may contribute to the stigmatization of the condition and other women's health problems. To address these issues, it is important for a comprehensive public health approach to be initiated to raise the awareness and educate the public about PCOS among women in Bangladesh.

\section{Acknowledgements}

The authors thank the volunteer respondents for their time and participation in the study.

\section{Conflict of interest}

All authors declare no conflicts of interest in this paper.

\section{References}

1. Sirmans SM, Pate KA (2013) Epidemiology, diagnosis, and management of polycystic ovary syndrome. Clinical Epidemiology 6: 1-13.

2. Allahbadia G, Merchant R (2008) Polycystic ovary syndrome in the Indian subcontinent. Seminars in Reproductive Medicine 26(1): 022-034.

3. Bruggmann D, Berges L, Kligelhofer D, et al. (2017) Polycystic ovary syndrome: Analysis of the global research architecture using density equalizing mapping. Reproductive Medicine Online 34: 627-638.

4. Gibson-Helm M, Teede H, Dunaif, A, et al. (2017) Delayed diagnosis and a lack of information associated with dissatisfaction in women with polycystic ovary syndrome. Journal of Clinical Endocrinology \& Metabolism 102: 604612.

5. Matsaseng, T (2017) Polycystic ovary syndrome: Management review and update. Obstetrics and Gynecology Forum 27: 1215.

6. Moran L, Gibson-Helm M, Teede H, et al. (2010) Polycystic ovary syndrome: A biopsychosocial understanding in young women to improve knowledge and treatment options. Journal of Psychosomatic Obstetrics and Gynecology 31(1): 24-31.

7. Williams T, Mortada R, Porter S (2016) Diagnosis and treatment of Polycystic Ovary Syndrome. American Family Physician 94(2).

8. McCook JG, Reame NE, Thatcher SS (2005) Health-Related quality of life issues in women with Polycystic Ovary Syndrome. Journal of Obstetric, Gynecologic \& Neonatal Nursing 34(1): 12-20.

9. McCook JG, Bailey BA, Williams SL, et al. (2015) Differential contributions of polycystic ovary syndrome (PCOS) manifestations to psychological symptoms. The Journal of Behavioral Health Services \& Research 42(3): 383-394.

10. Franks S (2017) Polycystic Ovary Syndrome. Medicine 45(9): 527-532.

11. Tan YM, Grigg J, Kulkarni J (2018) Borderline personality disorder and polycystic ovary syndrome: A review of the literature. Australian \& New Zealand Journal of Psychiatry 52(2): 117-128.

12. Thomson RL, Buckley JD, Lim SS, et al. (2010) Lifestyle management improves quality of life and depression in overweight and obese women with polycystic ovary syndrome. Fertility and Sterility, 94(5), 1812-1816.

13. Sills ES, Perloe M, Tucker MJ, et al. (2001). Diagnostic and treatment characteristics of polycystic ovary syndrome: descriptive measurements of patient perception and awareness from 657 confidential self-reports. BMC Women's Health 1(1): $1-5$.

14. Gibson-Helm M, Lucas IM, Boyle JA, et al. (2014) Women's experiences of polycystic ovary syndrome diagnosis. Family Practice 31(5): 545-549.

15. Dokras A, Saini S, Gibson-Helm M, et al. (2017) Gaps in knowledge among physicians regarding diagnostic criteria and management of polycystic ovary syndrome. Fertility and sterility 107(6): 1380-1386.

16. Tomlinson J, Letherby G, Pinkney, J, et al. (2010) Raising awareness of polycystic ovary syndrome. Nursing Standard 27(40): 35-39.

17. Avery JC, Braunack-Mayer AJ (2007) The information needs of women diagnosed with Polycystic Ovarian Syndrome implications for treatment and health outcomes. BMC Women's Health 7(1): 1-10.

18. Fatima P, Hossain MM, Rahman D, et al. (2016) Treatment seeking behavior and the profile of infertile patients attending the tertiary infertility center at Dhaka. Journal of Bangladesh College of Physician and Surgeons 10(3): 140-144.

19. Nahar, $P$ (2012) Invisible women in Bangladesh: Stakeholder's views on infertility services. Bangladesh Journal of Obstetrics and Gynecology 27(3): 30-37.

20. Nahrin NE, Ashraf F, Nessa K, et al. (2017) The emotionalpsychological consequences of infertility and its treatment. Bangladesh Journal of Obstetrics and Gynecology 32(2): 4244.

21. Rahman S, Chowdhury L, Khan R, et al. (2014) Hormonal evaluation of infertile women in Border Guard Hospital Peelkhana, Dhaka. Journal of Armed Forces Medical College Bangladesh 10(1): 34-48.

22. Saha A, Saifuddin K, Zaman F, et al. (2015) Mental health status of infertile women in Bangladesh. Universal Journal of Psychology 3(2): 51-54.

23. Vashakar SMK, Ehsan N, Hassen F (2016) Psychosocial effects of infertility among the childless couples at a specialized fertility center in Dhaka, Bangladesh. Bangladesh Journal of Obstetrics and Gynecology 31(1): 28-33. 
This work is licensed under Creative Commons Attribution 4.0 License

\section{To Submit Your Article Click Here: Submit Article}

DOI:10.31579/2642-9756/021
Ready to submit your research? Choose Auctores and benefit from:

* fast, convenient online submission

* rigorous peer review by experienced research in your field

* rapid publication on acceptance

* authors retain copyrights

* unique DOI for all articles

* immediate, unrestricted online access

At Auctores, research is always in progress.

Learn more https://www.auctoresonline.org/journals/women-health-careand-issues 